\title{
Path Planning for Robot Based on Improved Artificial Potential Field
}

\author{
Guoqing Qiu, Ting Niu, Qianqian Kou \& Cheng Liu \\ School of Electrical Engineering, Chongqing University of Posts and Telecommunications, Chongqing 400065, \\ China
}

\begin{abstract}
Aiming at the disadvantages of the traditional artificial potential field method, target is not reachable and the robot is easy to fall into local minimum point, the paper proposes an improved potential field function of artificial potential field, a formula factor is added to the repulsive force field function, with the closer the distance from the target point, the value of the factor decreases to zero, it can solve the defect of the target inaccessible, at the same time, In order to prevent robots from shocks and trapped in local minima, the paper adds the virtual target point in a small range from the target point to increase the attractiveness of the robot point to the target, so that the robot complete path planning, simulation results proved that the method is feasible and effective.
\end{abstract}

KEYWORD: artificial potential field, formula factors, virtual target point, path planning

\section{INTRODUCTION}

With the rapid development of the robot, more and more people are studying the robot, robot path planning is the main content of the research, which has been paid close attention to, the robot path planning algorithm has the traditional path planning method and intelligent method, for example, Genetic Algorithm ${ }^{[1]}$, Particle Swarm Optimization Algorithm ${ }^{[2]}$, Artificial Potential Field Method ${ }^{[3]}$ and so on, each algorithm has its own advantages and disadvantages, the Artificial Potential Field Method ${ }^{[4]}$ is widely usedbecause it is relatively simple and easily understand, but, it has the shortcomings that target inaccessible and trapped in local minima for its shortcomings, many researchers do the improvement, the literature[5] proposed narrowing the scope of obstacleclearance approach to improved artificial potential field method, the path planning of UAV(unmanned aerial vehicle) is carried out by using the improved gravity function of artificial potential field method in the literature[6],the optimal path search method that is an improved artificial potential field method combined with genetic algorithm is proposed in the paper[7],these methods can achieve better results, but there are still some problems.

To solve these problems, the paper improve the repulsive force field function based on the traditional artificial potential field method, the improved repulsive force field function is add a formula factors to avoid target inaccessible, in order to prevent the robot into a local oscillation, within the scope of the small target, assuming there have a virtual target point, when the robots trapped in local minima, the attraction of virtual target point for the robot will increase, robots can be out of minimum points, moving to the target point, and get the path planning of the robot.

\section{THE TRADITIONAL ARTIFICIAL POTENTIAL FIELD METHOD}

In 1985, the artificial potential field method was first proposed by Khatib, its basic idea is to assume that there is a virtual field, and construct potential field function, an unimpeded path is obtained in leading to the negative gradient direction of the potential field function ${ }^{[8]}$,regardless of quality and size of the robot, obstacles and target, we put them as a point, robots are repulsive to obstacles, the force between them is repulsive, the target point of the robot force is attractive, the target point is attractive to the robot, and the force between them is attractive, the robot is moving towards the target under the action of two force ${ }^{[9]}$, when the distance between the robot and the obstacle is getting smaller, the repulsive force will be greater, however, the closer the distance between the robot and the target, the smaller the attraction betweenthe target point and the robot, which is easy to cause the target is not reachable and fall into the local minimum points. 
The total potential energy function formula of traditional artificial potential field: $\vec{E}_{(p)}=\vec{E}_{a t t}(p)+\vec{E}_{r e p}(p)$, in this formula, the $\vec{E}_{\text {att }}(p)$ is the attractive potential energy function, the $\vec{E}_{\text {rep }}(p)$ is the repulsive potential energy function, their formula is as follows:

$$
\begin{aligned}
& \vec{E}_{\text {att }}(p)=\frac{1}{2} \times k \times \rho^{2}\left(p, p_{\text {goal }}\right) \\
& \vec{E}_{\text {rep }}(p)=\left\{\begin{array}{cc}
\frac{1}{2} \times \eta \times\left(\frac{1}{\rho^{2}\left(p, p_{\text {obs }}\right)}-\frac{1}{d_{0}{ }^{2}}\right) & \rho\left(p, p_{\text {obs }}\right) \leq d_{0} \\
0 & \rho\left(p, p_{\text {obs }}\right)>d_{0}
\end{array}\right.
\end{aligned}
$$

in the formula, $k$ and $\eta$ are positive proportion coefficient, $\rho\left(p, p_{\text {goal }}\right)$ is the distance between the robot and the target point, $\rho\left(p, p_{\text {obs }}\right)$ is the distance between the robot and the obstacles , $d_{0}$ is the radius of the robot, attraction is the negative gradient of attractive potential energy function, as shown in the formula (3):

$$
\vec{F}_{\text {att }}(p)=-\nabla \vec{E}_{\text {att }}(p)=k \times \rho\left(p, p_{\text {goal }}\right) \times \nabla \rho\left(p, p_{\text {goal }}\right)
$$

the repulsive force is the negative gradient of the repulsive potential energy function, as shown in the formula (4):

$$
\begin{aligned}
& \vec{F}_{\text {rep }}(p)=-\nabla \vec{E}_{\text {rep }}(p)= \\
& \left\{\begin{array}{cc}
\eta \times \frac{1}{\rho^{3}\left(p, p_{\text {obs }}\right)} \times \nabla \rho\left(p, p_{\text {obs }}\right) & \rho\left(p, p_{\text {obs }}\right) \leq d_{0} \\
0 & \rho\left(p, p_{\text {obs }}\right)>d_{0}
\end{array}\right.
\end{aligned}
$$

resultant force is what robot need, the attraction of the target point to the robot plus the sum of repulsive force between all obstacles and the robot, as shown in the formula (5):

$$
\vec{F}(p)=\vec{F}_{a t t}(p)+\sum_{i=1}^{n} \vec{F}_{r e p i}(p)
$$

\section{IMPROVED ARTIFICIAL POTENTIAL FIELD METHOD}

\subsection{Improved repulsive potential field function}

When the robot is about to reach the target point, the attraction between the target point and the robot is zero, however, the repulsive force between obstacles and the robot is likely to occur the phenomenon of non-zero, resulting in the appearance of the target unreachable, therefore, this paper improves the repulsive potential field, an exponential factor is added in the repulsive potential field function formula, with the decrease of the distance from the robot to the target point, it decreases and make the value of the repulsive potential field to zero at the target point, the improved repulsion potential field function formula as shown in (6):

$$
\vec{E}_{\text {rep }}(p)=\left\{\begin{array}{cl}
\frac{1}{2} \times \eta \times\left(\frac{1}{\rho^{2}\left(p, p_{o b s}\right)}-\frac{1}{d_{0}{ }^{2}}\right) \times\left(1-e^{\left(x-x_{g}\right)^{2}+\left(y-y_{g}\right)^{2}}\right) & \rho\left(p, p_{o b s}\right) \leq d_{0} \\
0 & \rho\left(p, p_{o b s}\right)>d_{0}
\end{array}\right.
$$

in the formula, $x, y$ is robot's coordinate value, $x_{g}, y_{g}$ is coordinate value of the target point, the calculation process of the repulsive force is shown as follows:

(1) Order $\mathrm{H}=\vec{E}_{\text {rep }}(p)$;

(2) Calculate $-\mathrm{H}_{x}^{\prime},-\mathrm{H}_{y}^{\prime}$;

$$
-\mathrm{H}_{x}^{\prime}=
$$$$
-\eta \times\left(x-x_{g}\right) \times\left(\frac{1}{\rho^{2}\left(p, p_{o b s}\right)}-\frac{1}{d_{0}^{2}}\right)^{2} \times e^{\left(x-x_{g}\right)^{2}+\left(y-y_{g}\right)^{2}}
$$

$+\eta \times\left(1-e^{\left(x-x_{g}\right)^{2}+\left(y-y_{g}\right)^{2}}\right) \times\left(\frac{1}{\rho^{2}\left(p, p_{o b s}\right)}-\frac{1}{d_{0}^{2}}\right) \times \frac{x-x_{g}}{\rho^{3}\left(p, p_{o b s}\right)}$

$-\mathrm{H}_{x}^{\prime}=-\eta \times\left(y-y_{g}\right) \times\left(\frac{1}{\rho^{2}\left(p, p_{\text {obs }}\right)}-\frac{1}{d_{0}{ }^{2}}\right)^{2} \times e^{\left(x-x_{g}\right)^{2}+\left(y-y_{g}\right)^{2}}$

$+\eta \times\left(1-e^{\left(x-x_{g}\right)^{2}+\left(y-y_{g}\right)^{2}}\right) \times\left(\frac{1}{\rho^{2}\left(p, p_{o b s}\right)}-\frac{1}{d_{0}^{2}}\right) \times \frac{y-x_{g}}{\rho^{3}\left(p, p_{o b s}\right)}$

(3) Calculate $\mathrm{H}=\sqrt{\left(-\mathrm{H}_{x}^{\prime}\right)^{2}+\left(-\mathrm{H}_{y}^{\prime}\right)^{2}}$
From the above process, we can be obtained when point targets near the robot and the resultant force of the robot is not zero, it can make the repulsive force to zero in the target point by using the improved repulsive potential field function to solve the shortcomings of destination unreachable.

\subsection{Virtual target artificial potential field method}

By using the traditional artificial potential field method, when the robot in the process of moving to point target, the attraction between target points and the robot and the repulsive force between obstacles and the robot balance, then the robot will in local minima, so this paper uses the method of increasing virtual target to make the force the robot bear unbalanced, and escape from local minima, the virtual target point is set at a $\varepsilon$ distance from the goal, and the range of $\varepsilon$ is very small, we can set any virtual target point in the range of 1, in Fig.1, it is the schematic diagram of the increased virtual target, 


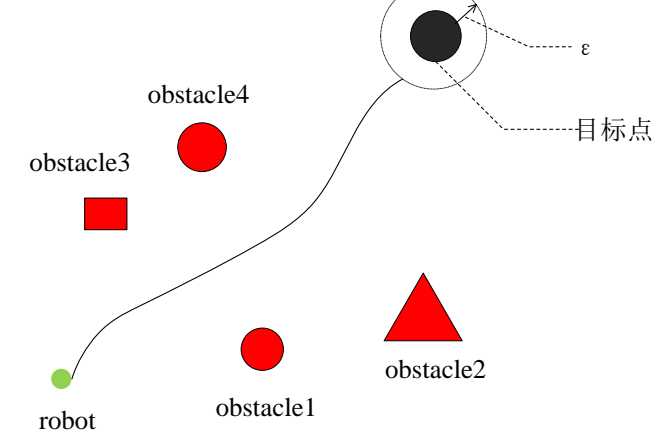

Fig. 1 Diagram of artificial potential field method of virtual target

In Fig.1, we can increase the virtual target point in the range of $\varepsilon$, robot's force is not zero, the robot break away from equilibrium point towards the target point.

\section{SIMULATION ANALYSIS ON IMPROVED ARTIFICIAL POTENTIAL FIELD METHOD}

\subsection{Improved artificial potential field method to avoid static obstacles}

Assuming the scope of the robot path planning is450 $\times 360$, within the scope, we set of a number of rectangular and triangular obstacles, and set the position coordinates of the robot and the target point, path simulation of traditional artificial potential field method and improved artificial potential field method are displayed in fig.2 and fig.3:

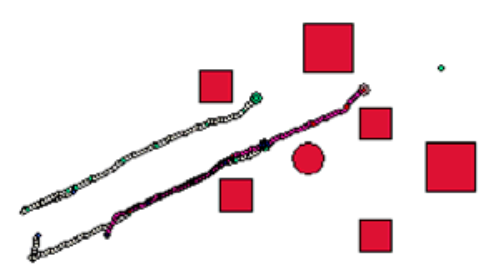

Fig.2 Simulation of traditional artificial potential field method

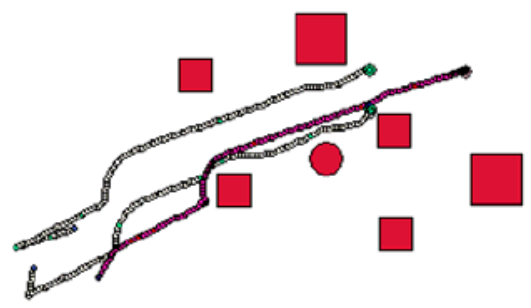

Fig.3 Simulation of improved artificial potential field method

From the two figures, the traditional artificial potential field method will appear target inaccessible and local turbulence, but the improved artificial potential field method can enable the robot to reach the target, and complete the route planning in absence of obstacles.

\subsection{Improved artificial potential field method to avoid dynamic obstacles}

In real life, not only do meet the robot static obstacles, but also it maybe meet the dynamic obstacles, Improved artificial potential field method can avoid dynamic obstacles effectively, the process of avoiding obstacle is divided into three parts, before obstacle avoidance, middle obstacle avoidance, and after obstacle avoidance, their simulation process as shown in figure 4:

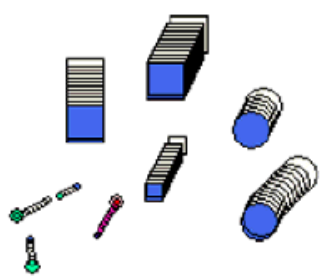

(a)

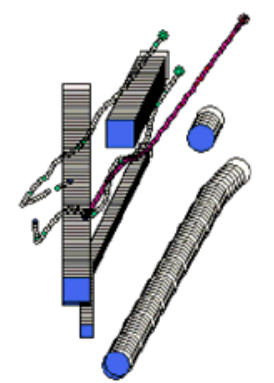

(c)

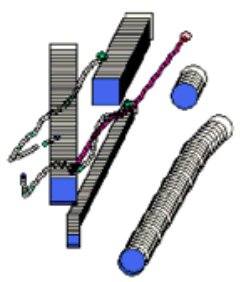

(b)
Fig.4 Simulation of dynamic obstacle avoidance based on improved artificial potential field method

From Fig. 3 shows that the blue circle and rectangle are dynamic obstacles, in Figure 4 (a), when the program began to run, the dynamic obstacles start moving, the robotrapidly change from the messy state to the set of triangles, in figure 4 (b), overlapping part is the trajectory of the moving obstacle, the robot encountered an obstacle,and began to avoid obstacle using improved artificial potential field method, its trajectory is presented irregular and bending ,because when the robot encounter the dynamic obstacle, it can quickly makeresponse, and select the other direction to avoid the dynamic obstacles,in figure4 (c), the robot to reach the target point, and avoid obstacle successfully.

\section{SUMMARY}

In this paper, the traditional artificial potential field method is easy to fall into the local minimum points and the target unreachable, so the improved artificial 
potential field method is presented, where the improvement is that the exponential factor is added in the repulsive potential energy function, this factor can make repulsive force change zero when the robot at the target point, which can solve the problem that the robot can not reach the goal, In addition, the addition of virtual target can avoid that the force of the robot is zero, and make it in a state of equilibrium on the basis of this, the simulation results show that the proposed algorithm is feasible and effective.

\section{REFERENCES}

[1] Liu Guodong, Xie Hong-bin, Li Chunguang. Method of mobile robot path planning in dyamic environment based on genetic algorithm[J]. Robot, 2003, 327-331.

[2]Liu Guanjun. Research of path planning for mobile robots based on Particle Swarm Optimization[D].Central South University, 2007.

[3] O.Khatib. Real time obstacle avoidance for manipulators and obile robots[J].IEEE International conference on Robotics andAutomation.1985,500-505.

[4] Zhuang Huizhong, Du Shuxin, Wu Tiejun, Research on path planning and related algorithms for robots[J]. Bulletin of science and technology, 2004, 20 (3): 210 -215.

[5]Guo Guangyao, Wen Sufang.Path planning of mobile robot based on improved artificial potential field approach[J]. Computer engineering and design, 2015, 36(10):2819-2822.

[6]Ding Jiaru, Du Changping, Zhao Yao, etc. Path planning algorithm for unmanned aerial vehicles based onimproved artificial potential field[J]. Journal of Computer Applications, 2016, 36(1):287-290.

[7]Kuang Fei, Wang Yaonan.Robot path planning based on Hybrid Artificial Potential Field / Genetic Algorithm[J].Journal of System Simulation, 2006,18(3):774-777.

[8]Zhang Huanghui. Research and application path planning based on dynamic artificial potential field[D].Chang sha: Chang sha University of science \& Technology,2010.

[9] Li Zhou, Wei Li. Adaptive artificial potential field approachfor obstacle avoidance path planning[A].IEEE ComputerSociety[C].2014,429-432. 